Z. klin. Chem. u. klin. Biochem.

7. Jg., S. 444-447, September 1969

\title{
Eine empfindliche Methode zur Bilirubinbestimmung
}

\author{
Von H. Bartels und M. BöHMER \\ Aus dem Chemischen Laboratorium des Bürgerspitals Basel
}

(Eingegangen am 8. April 1969)

Es wird eine Methode zur Bilirubinbestimmung beschrieben, welche als Diazokomponente das stabilisierte 2-Chlor-4-nitroanilin verwendet. Gekuppelt wird in saurem Milieu in Gegenwart von Ultravon als Löslichkeitsvermittler. Die Technik ist so konzipiert, daß lediglich eine Verdünnung und eine Pipettierung notwendig sind. Die Reagenzien sind stabil und preiswert, der Leerwert kann für Routinezwiecke vernachlässigt werden.

\section{A sensitive method for the determination of bilirubin}

A method is described for the determination of bilirubin, which employs the stabilised 2-chloro-4-nitroaniline as the diazo component. Coupling is performed under acidic conditions in the presence of ultravone as the solubilising agent. The operation is designed so that only one dilution and one transfer by pipette are necessary. The reagents are stable and economical. For scanning purposes the blank may be ignored.

Die Kenntnis der Bilirubinkonzentration im Serum ist auch heute noch ein wichtiges Indiz zur Beurteilung von Schwere und Verlauf der verschiedenen Ikterusformen. Im klinisch-chemischen Routinelabor wird sie durch die photometrische Messung des Kupplungsproduktes mit diazotierter Sulfanilsäure bestimmt. Unter Abspaltung eines Carboniumions reagiert das Diazoniumsalz in zwei Schritten zu einem Azofarbstoff mit Indikatoreigenschaften: im stark sauren Bereich ist er blau, im neutralen rot und bei alkalischer Reaktion wieder blau (1).

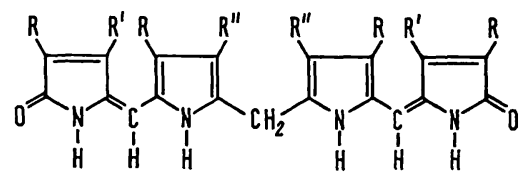

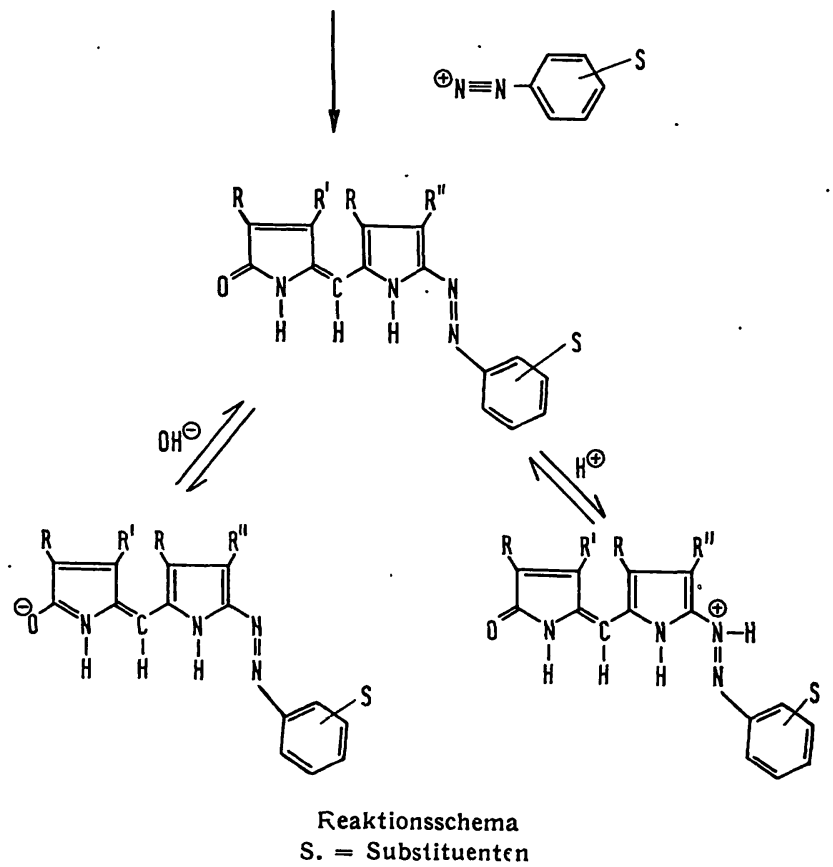

Der molare Extinktionskoeffizient $\varepsilon$ des Reaktionsproduktes ist - bei der am meisten verwendeten Methode, welche im alkalischen Bereich mißt - am Maximum um $600 \mathrm{~nm}$ nur etwa $6,9 \cdot 10^{4} . \mathrm{cm}^{2} / \mathrm{Mol}(2)$; man muß daher mit einer geringen Serumverdünnung arbeiten. Außerdem ist die Extinktion des Leerwertansatzes bei dieser Wellenlänge unter anderem von der Bilirubinkonzentration abhängig. Die Methode bedingt eine ganze Anzahl von Pipettierungen, die Diazokomponente muß täglich frisch zubereitet werden.

\section{Fragestellung}

Unter Beibehaltung des seit über 80 Jahren bewährten Reaktionsmechanismus wollten wir eine 'rationelle Technik entwickeln, die es dank eines höheren Extinktionskoeffizienten des Kupplungsproduktes erlauben sollte, weniger Serum einzusetzen; möglicherweise würde sich der Leerwert erübrigen. Neben einem hohen Extinktionskoeffizienten des Kupplungsproduktes sind für die Diazokomponente noch folgende Kriterien zu erfüllen:

1. Sie muß sehr leicht zugänglich sein, sowie - auch in Lösung - über längere Zeit stabil bleibèn.

2. Die Kupplungsreaktion soll schnell ablaufen, damit keine Wartezeiten auftreten.

3. Die maximale Exxtinktion soll bei einer Wellenlänge liegen, bei der der Leerwert eine minimale Extinktion aufweist.

Versucht wurde, die Sulfanilsäure durch andere substituierte Anilinderivate bzw. durch im Handel befindliche, stabilisierte Diazoniumsalze zu ersetzen. Hier kommen besonders solche Substituenten in Frage, welche einen elektrophilen Charakter haben. Einerseits lassen sie eine Farbvertiefung érwarten und zudem eine schnelle Kinetik, da die positive Ladung am Diazostickstoff erhöht wird. Des weiteren versuchten wir, die 
in der Farbstoffchemie oft benützte Fähigkeit gewisser Azofarbstoffe, Metallkomplexe zu bilden, analytisch auszuwerten. Das diazotierte Bilirubin hat ebenfalls eine für Komplexbildung günstige Struktur:

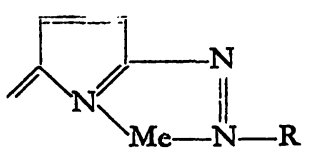

Unter Beteiligung des Stickstoffs im Pyrolkern können Chelate gebildet werden.

\section{Ergebnisse}

In Tabelle 1 sind die untersuchten Anilinderivate mit den entsprechenden Wellenlängen der maximalen Absorption und dem molaren Extinktionskoeffizienten zusammengestellt. Es kam uns hier nicht darauf an, Präzisionsmessungen durchzuführen, sondern einen Eindruck von der Variabilität der Farbtiefe und des Farbtons als Funktion der Substituenten zu bekommen.

Tab. 1

Extinktionskoeffizienten von verschiedenen Diazobilirubinen

\begin{tabular}{lccc}
\hline \multicolumn{1}{c}{ Anilinderivat } & $\lambda \max (\mathrm{nm})$ & $\epsilon\left(\mathrm{cm}^{2} / \mathrm{Mol}\right)$ & $\mathrm{pH}$ \\
\hline 2-Chloranilin & 590 & $0,65 \cdot 10^{8}$ & 11,8 \\
2-Jodanilin & 570 & $0,24 \cdot 10^{5}$ & 11,8 \\
\hline 3-Jodanilin & 590 & $0,32 \cdot 10^{5}$ & 11,8 \\
\hline 4-Chloranilin & 590 & $0,24 \cdot 10^{5}$ & 11,8 \\
4-Jodanilin & 590 & $0,35 \cdot 10^{5}$ & 11,8 \\
\hline 2,4-Dichloranilin & 600 & $0,70 \cdot 10^{8}$ & 11,8 \\
2-Chlor-4-nitroanilin & 660 & $0,91 \cdot 10^{5}$ & 11,8 \\
2,4-Dibromanilin & 590 & $0,61 \cdot 10^{5}$ & 11,8 \\
2,5-Dibromanilin & 590 & $0,52 \cdot 10^{5}$ & 11,8 \\
-2,4,5-Trichloranilin & 600 & $0,71 \cdot 10^{5}$ & 11,8 \\
Sulfanilsäure & 605 & $0,69 \cdot 10^{5}$ & 11,8 \\
Echtrotsalz B & 665 & $0,78 \cdot 10^{5}$ & 11,9 \\
Echtblausalz BB & 620 & $1,00 \cdot 10^{\circ}$ & 11, \\
\hline
\end{tabular}

2-Chlor-4-nitro-anilin und Echtblausalz BB bewirken einen intensiven bathochromen Shift der Absorptionsbande und weisen aụch einen hohen molaren Extinktionskoeffizienten auf. Die Reaktion ist jedoch für Echtblausalz BB sehr heikel, da die Kupplung erst oberhalb von $\mathrm{pH} 7$ genügend schnell abläuft, wo das Reaktionsprodukt der Autoxydation unterliegt und schnell ausbleicht.

Versuche, die Komplexbildung mit Metallsalzen zur Farbvertiefung heranzuziehen, schlugen fehl: Zwar reagieren Kupfer-, Nickel- und Kobaltionen zu schön gefärbten Komplexsalzen, und es tritt auch eine bathochrome Verschiebung der Absorptionsbande auf, aber gleichzeitig eine Verbreiterung. Der Extinktionskoeffizient ist niedriger.

Es war von Interesse, den Einfluß der Schwermetallionen des Serums auf die Nachwieisreaktion des Bilirubins zu untersuchen. Wäßrige Bilirubinlösungen wurden in Gegenwart von physiologisch möglichen Metallionenkonzentrationen gekuppelt: $500 \mu \mathrm{g} / 100 \mathrm{ml}$ an $\mathrm{Cu}, \mathrm{Fe}$ und $\mathrm{Co}$ haben keinen meßbaren Einfluß auf

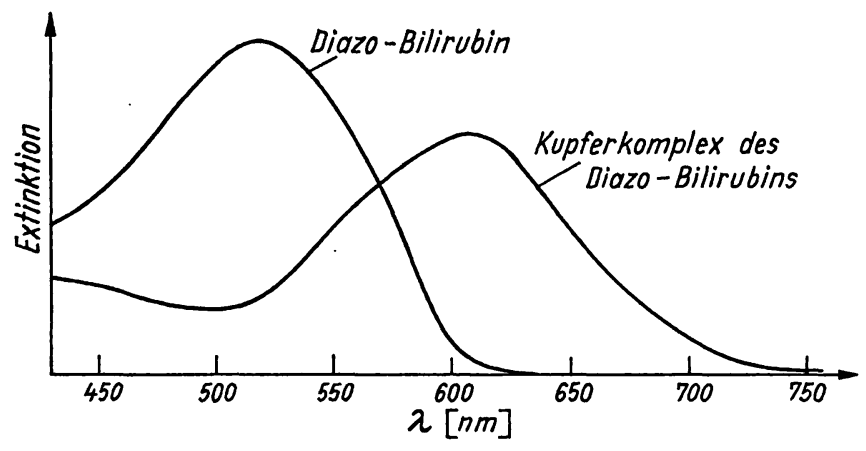

Abb. 1

Spektrum des Kupferkomplexes von Diazobilirubin $5 \mathrm{ml}$ Harnstoff/Na-Benzoat

$0,5 \mathrm{ml}$ Bili-Control $20 \mathrm{mg} / 100 \mathrm{ml}$

$0,25 \mathrm{ml}$ diazotierte Sulfanilsäure

pH 6,6 Sulfanilsäure $0,01 \mathrm{M}$ in $0,25 \mathrm{M} \mathrm{HCl}+0,1 \mathrm{ml} \mathrm{NaNO}_{2} 0,1 \mathrm{M}$ )

$0,2 \mathrm{ml} \mathrm{CuSO} \cdot 5 \mathrm{H}_{2} \mathrm{O} 100 \mathrm{mg} / 100 \mathrm{ml}$

die Absorptionsbande des mit diazotierter Sulfanilsäure und Bilirubin erhaltenen Farbstoffes. Die weiteren Experimente wurden mit 2-Chlor-4-nitroanilin durchgeführt. Diese Verbindung läßt sich wegen der in verdünnter Säure geringen Löslichkeit nicht so leicht diazotieren, wie z. B. Sulfanilsäure. Aus diesem Grunde sahen wir davon $a b$, die Diazotierung täglich auszuführen. Wir stellten das entsprechende Diazoniumsalz dar und brachten es mit Naphthalin-1,5-disulfosäure zur Fällung. Dieses stabilisierte Salz ist sehr gut löslich und in saurem Milieu auch über mehrere Wochen (wir prüften bis zu sechs Wochen) genügend stabil, solange kein Licht Zugang hat. Diese Tatsache veranlaßte uns, die Kupplung bei saurem $\mathrm{pH}$ vorzunehmen, was -weiterhin den Vorteil hat, daB das Bilirubin Oxydationsstabil ist. Die Elektrophilie der Diazogruppe reicht aus, um bei einem $\mathrm{pH}$ von $\sim 2$ eine hinreichend schnelle Kinetik der Kupplung zu erhalten.

Schwierig ist hingegen, das nicht glucuronidierte Bilirubin bei stark saurem $\mathrm{pH}$ in Lösung zu bringen, so daß die Farbbildung trotzdem nur sehr langsam abläuft. Als Löslichkeitsvermittler bei der Kupplung im Neutralbereich werden verschiedene Substanzen zugesetzt. Benzoesäure ist aber bei saurem $\mathrm{pH}$ nicht löslich, Diphyllin oder Coffein unwirksam, Methanol bewirkt Trübungen. HirlumanN (3) schlägt die Verwendung von Äthylenglykol vor, das aber wegen der hohen Viskosität unpraktisch ist. Mischungen aus Methanol und Äthylenglykol stellen einen Kompromiß dar. Noch befriedigendere Resultate erhält man bei Verwendung von Dispersionsmitteln, die in der präparativen industriellen Chemie verwendet werden. Wir haben eine Reihe dieser bezüglich ihrer akzelerierenden Wirkung auf die Farbbildung untersucht und Ultravon $\mathrm{JF}^{1}$ ), Neolansalz $\mathrm{P}^{1}$ ) und Teepol ${ }^{2}$ ) als sehr wirksam befunden. Bei Verwendung von Teepol treten jedoch hin und wieder Niederschläge auf. Die beiden übrigen Substanzen beschleunigen die Umsetzung von diazotiertem 2-Chlor-4-nitroanilin und Bilirubin / Albumin (Bili-Control Merz \&

1) Ciba AG, Bascl.

2) Shell Corp. 
Dade) bei pH 1,8 und einer Konzentration von $10 \mathrm{mg} /$ $100 \mathrm{~m} l$ des Ansatzes so stark, daß nach 3 Minuten bereits $>98 \%$ umgesetzt sind.

Bezüglich der differenzierten Bestimmung des glucuronidierten Bilirubins - dem "direkt reagierenden" - ist zu bedenken, daß bei neutralem oder gar alkalischem $\mathrm{pH}$ das unglucuronidierte Bilirubin ebenfalls in Lösung geht und schneller diazotiert wird. Die Erfassung der glucuronidierten Verbindung sollte daher mit einer möglichst schnell kuppelnden Diazokomponente geschehen. $\mathrm{Da}$ die Reaktionsordnung der Farbreaktion komplexer Natur ist, lassen sich keine Geschwindigkeitskonstanten berechnen. Qualitative Versuche, bei denen, wie herkömmlich die Extinktion nach 1 Minute als $\mathrm{Ma} \beta$ genommen wurde, machen wahrscheinlich, daß z. B. das 2,4-Dichloranilin und 2-Chlor-4-nitro-anilin der Sulfanilsäure überlegen sind, indem sie bei gleichem $\mathrm{pH}$ um einiges schneller reagieren.

Um herauszufinden, bei welcher Wellenlänge der Serumleerwert am kleinsten ist, nahmen wir die Spektren einer Reihe verdünnter Seren auf. Ansätze, welche mit Ultravon/Salzsäure und Fehling II-Lösung - in der Art wie unter „Methodisches" angegeben - verdünnt wurden, zeigten, daß die Lösungen oberhalb von $640 \mathrm{~nm}$ nahezu optisch leer waren, so daß eine Messung mit Vorteil hier ausgeführt wird.

\section{Methodisches}

\section{Reagenzien}

Die Reagenzien stammen, wenn nichts anderes erwähnt, von Merck A.G. Darmstadt oder Fluka Buchs/SG (Schweiz). Das Diazo-2-chlor-4-nitro-anilin wurde selbst hergestellt. Falls die Substanzen nicht chromatographisch rein waren, wurden sie umkristallisiert, bis dieses Kriterium zutraf.

Darstellung des stabilisierten Diazoniumsalzes von 2-Chlor-4-nitro-anilin

$\mathrm{Zu} 30 \mathrm{~m} /$ demineralisiertem Wasser gibt man $1 \mathrm{~g}$ Dispersionsmittel (Ultravon W, Brandoran A), $60 \mathrm{~g} 33$ proz. Salzsäure und innerhalb 15 Minuten unter Rühren $31,6 \mathrm{~g}$ fein pulverisiertes, getrocknetes 2-Chlor-4-nitroanilin. Nachdem eine homogene Dispersion erhalten wurde, wird $100 \mathrm{~g}$ fein gestoßenes Eis zugesetzt, wonach die Temperatur auf $-8^{\circ}$ fällt. Nunmehr werden unter die Flüssigkeitsoberfläche (!) $32,5 \mathrm{~m} l 40$ proz. $\mathrm{NaNO}_{2}-$ Lösung innerhalb 5 Minuten zugegeben. Die ablaufende Reaktion wärmt die Lösung auf $7-8^{\circ}$ auf. Nachdem 30 Minuten gerührt wurde, ist die Base fast restlos in Lösung gegangen. Den filtrierten Ansatz tropft man unter Rühren in eine konzentrierte Lösung von Naphthalin-1,5-disulfosäure. Der rot-orangene Niederschlag wird abfiltriert und im Vakuum getrocknet.

Reagenz-Lösungen

Diazolösung 0,106 mм 2-Chlor-4-nitro-anilin.

$50 \mathrm{mg}$ des stabilisierten Diazo-2-chlor-4-nitro-anilins werden mit $100 \mathrm{~m} / 1 \mathrm{~N} \mathrm{HCl}$ und $10 \mathrm{~m} l$ Ultravon gelöst und mit demineralisiertem Wasser auf $1000 \mathrm{ml}$ aufgefüllt. (Im Dunkeln bei $4^{\circ}$ mindestens 1 Monat haltbar.)

Febling II-Lösung Tartrat $0,3725 \mathrm{M}, \mathrm{NaOH} 0,625 \mathrm{~N}$.

$105 \mathrm{~g}$ Kalium-Natriumtartrat und $25 \mathrm{~g}$ Natrium-hydroxid werden auf $1000 \mathrm{ml}$ aufgefüllt (unbeschränkt haltbar).

\section{Meßansatz}

$0,1 \mathrm{~m} l$ Serum wird in $1 \mathrm{~m} l$ Diazolösung pipettiert und nach 3 Minuten mit $1 \mathrm{~m} l$ Fehling II-Lösung versetzt. Gemessen wird bei 660-680 nm oder bei der Hg-Linie $691 \mathrm{~nm}$.

\section{Diskussion der Methode}

Die ausgearbeitete Methode benützt das stabilisierte Diazoniumion des 2-Chlor-4-nitro-anilins als Kupplungskomponente. Die Reaktion findet bei $\mathrm{pH} 2$ statt, wobei das unkonjugierte Bilirubin durch Ultravon in Lösung gebracht wird. Anschließend wird alkalisch gestellt, so $\mathrm{da} \beta$ die Lösung von rot nach blau umschlägt. Eine Standardkurve, welche aus einer Bilirubin/AlbuminLösung verdünnt wird, gehorcht dem LAMBERTBEERschen Gesetz bis zu einer Konzentration von $15 \mathrm{mg} / 100 \mathrm{~m} l$ Bilirubin. Störende Substanzen konnten wir nicht finden. In saurem Ansatz beruht das Ausmaß der roten Farbe zum größten Teil auf der Konzentration des Kupplungsproduktes mit Bilirubin. Der Rest geht auf Reaktionsprodukte mit Histidin und Tyrosin zurück, die aber oberhalb pH11 nicht in eine blaue Farbkomponente umschlagen. Mit einer reinen Albuminlösung von $8 \mathrm{~g} / 100 \mathrm{~m} l$ findet man eine Extinktion für $\mathrm{pH} 11$ bei einer Wellenlänge um $660 \mathrm{~nm}$, welche etwa $100 \mu \mathrm{g} / 100 \mathrm{~m} l$ Bilirubin vortäuscht. Das gleiche gilt für den größten Teil der Seren. Lediglich bei sehr stark lipämischen Proben läßt sich ein größerer Leerwert messen: für Seren mit der Extinktion von 1,4 (bei $600 \mathrm{~nm}$ ) fanden wir Leerwerte, die eine Konzentration von $0,4 \mathrm{mg} / 100 \mathrm{ml}$ Bilirubin vortäuschen. Das zugesetzte Ultravon vermag nicht nur das Bilirubin in Lösung zu bringen, sondern auch einen Teil des Fetts zu dispergieren. Im folgenden führen wir einige Kriterien an, welche die Brauchbarkeit der beschriebenen Technik demonstrieren sollen:

Ein wichtiges Argument ist die Reprodurierbarkeit der gefundenen Werte. Diese schätzen wir ab, indem Doppelbestimmungen in je zwei verschiedenen Serien durchgeführt wurden. Um den „psychologischen Effekt" bei der Ablesung auszuschalten, wurde nicht ein bestimmtes Serum, sondern täglich ein anderes, das jeweils am Vortage analysiert worden war, in die Serie gegeben. Aus der graphischen Darstellung (Abb. 2) ist ersichtlich, daß die auftretenden Fehler über einen weiten Konzentrationsbereich $\pm 3 \%$ nicht überschreiten, was für klinische Zwecke voll ausreichend ist.

Eine Vorstellung über die Speqifität der Methode wurde gewonnen, indem mit einer Referenzmethode Vergleichswerte erhalten wurden. Als Referenzmethode 


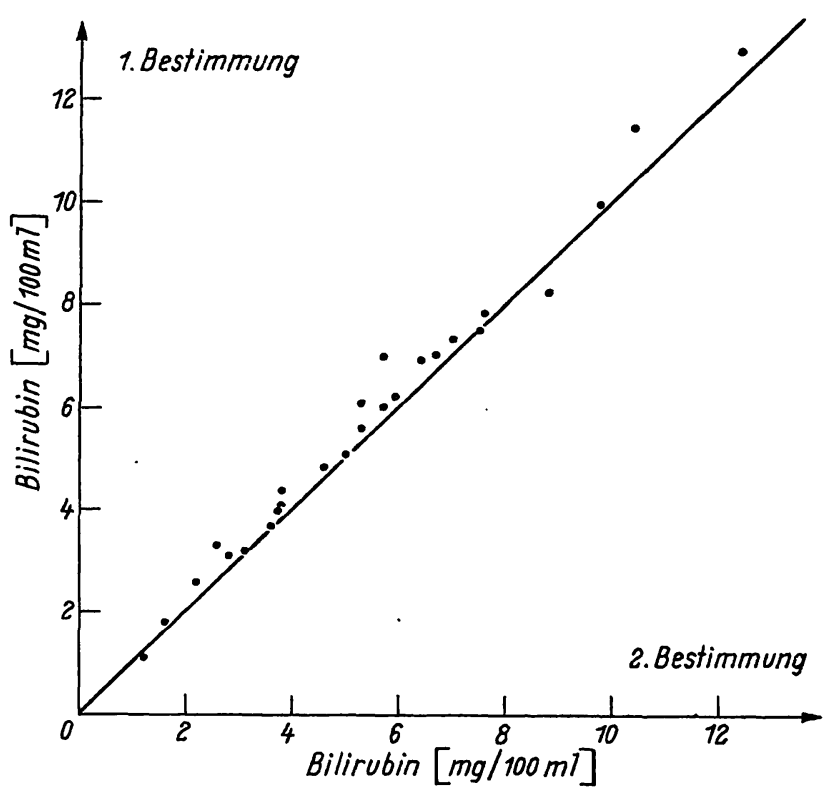

Abb. 2

Reproduzierbarkeit der Bilirubinbestimmung unter Routinebedingungen an zwei aufeinanderfolgenden Tagen.

wählten wir die von Mrchaelsson (4). Da die Bilirubinwerte innerhalb des Normalbereiches nur von untergeordneter Wichtigkeit sind, wurden nur erhöhte Konzentrationen verglichen. Die Ergebnisse sind in Abbildung 3 dargestellt. Die Gegenüberstellung beweist,

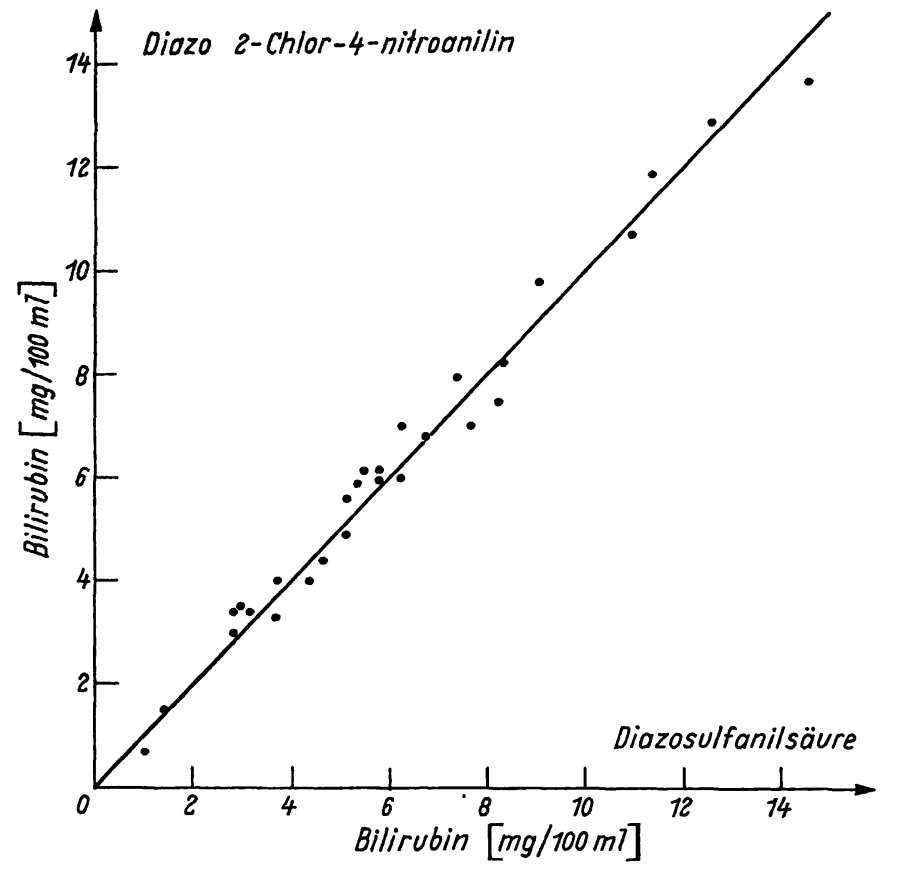

Abb. 3

Darstellung von Wertepaaren, die einerseits mit der hier beschriebenen Methode und andererseits mit der klassischen Sulfanilsäure-Technik erhalten wurden.

daß die Arbeitshypothese richtig war. Der gleiche Reaktionsmechanismus ergibt weitgehend identische Konzentrationen, so daß die aus der Erfahrung gewach-. senen klinischen Interpretationsmöglichkeiten der Werte erhalten bleiben.

\section{Literatur}

1. Billing, B. H., P. G. Cole und G. M. Zathe, Biochem. J. 65, manN, G. und G: Berger, diese Z. 5, 92 (1967). - 4. Micha774 (1957). - 2. LaUber, K., diese Z. 5, 278 (1967). - 3. Hrll- elsson, M., Scand. J. clin. Laborat. Invest. 13, 56 (1961).

Dr. H. Bartels

Bürgerspital

$\mathrm{CH} 4000$ Basel 